\title{
SRT1720, a SIRT1 activator, promotes tumor cell migration, and lung metastasis of breast cancer in mice
}

\author{
KENSUKE SUZUKI $^{1}$, RYUJI HAYASHI ${ }^{1}$, TOMOMI ICHIKAWA ${ }^{1}$, SHINGO IMANISHI ${ }^{1}$, TORU YAMADA ${ }^{1}$, \\ MINEHIKO INOMATA ${ }^{1}$, TOSHIRO MIWA ${ }^{1}$, SHOKO MATSUI $^{1}$, ISAO USUI ${ }^{1}$, MASAHARU URAKAZE $^{1}$, \\ YUJI MATSUYA ${ }^{2}$, HIROFUMI OGAWA ${ }^{2}$, HIROAKI SAKURAI ${ }^{3}$, IKUO SAIKI $^{3}$ and KAZUYUKI TOBE ${ }^{1}$ \\ ${ }^{1}$ First Department of Internal Medicine, ${ }^{2}$ Laboratory of Synthetic Medicinal Chemistry, Graduate \\ School of Medicine and Pharmaceutical Sciences and ${ }^{3}$ Division of Pathogenic Biochemistry, \\ Institute of Natural Medicine, University of Toyama, Toyama 930-0194, Japan
}

Received December 29, 2011; Accepted February 22, 2012

DOI: $10.3892 /$ or.2012.1750

\begin{abstract}
Silent information regulator 2 (SIR2) is a highly conserved protein, the mammalian orthologue of which, SIRT1, exhibits histone deacetylase activity. SIRT1 is involved not in only longevity due to caloric restriction but in a variety of diseases such as diabetes, cardiovascular dysfunction and neurodegeneration. However, accumulating evidence shows that SIRT1 is overexpressed in various types of malignant cells, and its inhibitors suppress the growth of tumor cells. The relationship between SIRT1 and metastasis remains to be clarified. Here, we examined the effect of SRT1720, a SIRT1 activator, on lung metastasis of breast cancer cells. 4T1 breast cancer cells were subcutaneously implanted into syngeneic BALB/c mice and SRT1720 was administered alone or with an antitumor agent, cisplatin. As expected, cisplatin decreased the lung metastasis score, whereas SRT1720 increased metastasis irrespective of cisplatin. In the primary tumors, cisplatin suppressed the mRNA level of angiopoietin-like protein 4 (ANGPTL4), a lung metastasis-promoting gene product of breast cancer, but SRT1720 reduced the effectiveness of cisplatin. The results obtained with animal experiments were in accordance with those in human cancer cells; SRT1720 significantly increased the amount of VEGF secreted from MDA-MB-231 cells. Moreover, a transendothelial cell migration assay showed that SRT1720 promotes the migration of MDA-MB-231 cells across an endothelial cell layer despite the presence of cisplatin. These findings imply that SRT1720 promotes the pulmonary metastasis of breast cancer cells and SIRT1 may be an important target for suppressing metastasis to the lung.
\end{abstract}

Correspondence to: Dr Ryuji Hayashi, First Department of Internal Medicine, University of Toyama, 2630 Sugitani, Toyama 930-0194, Japan

E-mail: htaro@med.u-toyama.ac.jp

Key words: SIRT1, SRT1720, breast cancer, metastasis, migration, murine model

\section{Introduction}

Metastasis is a poorly understood process in cancer biology despite extensive study (1). It comprises multiple steps; movement of cells from the primary tumor into the surrounding tissues, penetration of blood and lymphatic vessels, extravasation into the organ parenchyma, and proliferation to form metastatic colonies at secondary sites. These steps require the coordinated actions of numerous genes $(2,3)$. Several studies have recently clarified that cancer cells regulate the expression of specific genes involved in the targeted colonization of other organs; these genes include an 18-gene breast-to-lung metastatic gene-expression signature, such as angiopoietinlike protein 4 (ANGPTL4), epidermal growth factor receptor ligand epiregulin, prostaglandin-endoperoxide synthase 2 (also known as COX2), matrix metalloproteinase-1, and other mediators associated with infiltration of cancer cells and subsequent colonization in the lung $(4,5)$.

Silent information regulator 2 (SIR2) is a highly conserved protein found from yeast to humans. The mammalian Sirtuin family is comprised of seven members (6) and SIRT1 is the mammalian SIR2 orthologue which exhibits histone deacetylase activity (HDAC). It is well established that caloric restriction expands lifespan in a variety of species, in which SIRT1 plays the role of a principal modulator (7-9). On the other hand, SIRT1 expression is upregulated in malignant cells or tissues from patients with leukemia, glioblastoma, and prostate, colorectal, skin cancers, and therefore, SIRT1 is considered to have a pivotal role in tumor initiation, progression and drug resistance by blocking senescence and apoptosis or promoting cell growth (10). Furthermore, a specific SIRT1 inhibitor such as sirtinol impedes cell growth, increases the sensitivity to drugs of androgen-refractory prostate cancer cells (11), and induces senescence-like growth arrest in both human breast cancer MCF-7 cells and lung cancer H1299 cells (12). Another SIRT1 inhibitor, cambinol, reduces tumor growth in animal models (13), and tenovin-6, an inhibitor of both SIRT1 and SIRT2, decreases tumor growth in xenografted human melanoma cells (14). Conversely, resveratrol, a naturally occurring polyphenol abundant in red wine, was identified as a promising compound for cancer treatment 
through the probable activation of SIRT1 $(15,16)$. Recently, SRT1720, a newly synthesized compound, has been identified as a specific activator of SIRT1 (17). The activity of SRT1720 as a SIRT1 activator is stronger than that of resveratrol. We previously reported that SRT1720 treatment decreased the expression of marker genes responsible for oxidative stress and inflammatory cytokines in the liver of monosodium glutamate (MSG)-injected ICR mice, a murine model of severe obesity and insulin resistance (18).

While SIRT1 expression is upregulated in various cancers and SIRT1 inhibitors suppress cancer cell growth in vivo and in vitro, the relationship between the function of SIRT1 and cancer metastasis remains to be characterized. In this study, we investigated the effects of SRT1720 on lung metastasis of breast cancer cells in mice as well as cultured murine and human breast cancer cells, and concluded that SRT1720 deduces metastasis.

\section{Materials and methods}

Reagents. SRT1720 was chemically synthesized by the method of Milne et al (17). The structure and purity of the compound were confirmed by NMR spectroscopy. The protein-deacetylating activity of SRT1720 was checked with a CycLex SIRT1/ Sir2 deacetylase fluorometric assay kit (Biomol International LP, Plymouth Meeting, PA, USA). Cisplatin (Wako Pure Chemical Industries, Ltd., Tokyo, Japan) was dissolved in sterile water and kept at $-20^{\circ} \mathrm{C}$ until used. Other chemicals were commercially available.

Mice. BALB/c mice (5-week-old females) were purchased from Japan SLC (Hamamatsu, Shizuoka, Japan). The mice were maintained under laminar airflow conditions with a 12 h-light (6:00-18:00)/12 h-dark (18:00-6:00) cycle. Laboratory chow and water were freely available. This study was performed in accordance with the guidelines for the care and use of laboratory animals of the University of Toyama.

Cells. 4T1 cells, a type of spontaneously metastasizing murine mammary adenocarcinoma cells (CRL-2539), were obtained from the American Type Culture Collection (ATCC, Manassas, VA, USA) and passaged for fewer than 6 months. The cells were cultured to a monolayer in Dulbecco's modified Eagle's medium (Life Technologies/Gibco-BRL, Paisley, UK) containing $10 \%$ fetal bovine serum, sodium pyruvate, non-essential amino acids, L-glutamine and vitamins, and 10 mM HEPES buffer (pH 7.4). MDA-MB-231 cells, a human breast cancer cell line, were from the European Collection of Cell Cultures (ECACC, Salisbury, UK) and were passaged for fewer than 6 months. They were cultured in Leibovitz's L-15 medium (Gibco-BRL) containing $10 \%$ fetal bovine serum, sodium pyruvate, non-essential amino acids, L-glutamine and vitamins. The 4T1 cells were maintained in $75-\mathrm{cm}^{2}$ ventilated flasks in air containing $5 \% \mathrm{CO}_{2}$ at $37^{\circ} \mathrm{C}$, while the culture conditions for the MDA-MB-231 cells were the same as those of the $4 \mathrm{~T} 1$ cells except for $0.2 \% \mathrm{CO}_{2}$. Primary human umbilical vein endothelial cells (HUVECs) were from Cell Applications (San Diego, CA, USA), and were not passaged for more than 6 months.
Cell viability assay. One day before treatment, $1.5 \times 10^{4}$ cells per well were seeded in a 96-well plate and allowed to attach overnight. The cells were then treated with varying concentrations of SRT1720 for $24 \mathrm{~h}$. The viable cell number was determined with an MTS assay kit (Promega, Madison, WI, USA).

Confocal microscopy. 4T1 cells were incubated on a 4-chamber polystyrene vessel tissue culture slide (Becton-Dickinson, Franklin Lakes, NJ, USA) for 2 days, and fixed in $4 \%$ paraformaldehyde phosphate buffer for $15 \mathrm{~min}$, followed by treatment with $0.1 \%$ Triton $\mathrm{X}-100$ for $10 \mathrm{~min}$ to improve permeability. After washing, the cells were blocked with $10 \%$ normal goat serum in PBS containing 0.1\% Triton X-100 for $60 \mathrm{~min}$, and treated with a primary anti-SIRT1 rabbit antibody (Millipore, CA, USA) [diluted 1:200 with PBS containing 1\% bovine serum albumin (BSA)] for $2 \mathrm{~h}$. After three washes with PBS, the cells were incubated with an Alexa Fluor 594 anti-rabbit IgG (diluted 1:750 with 1\% BSA) (Life Technologies, Tokyo, Japan) for $30 \mathrm{~min}$. After three more washes with PBS, the cells were incubated with $0.2 \mu \mathrm{M} 4$ ',6-diamidino-2-phenylindole (DAPI) (Life Technologies) in PBS for $1 \mathrm{~min}$ and washed. The slides containing the treated cells were filled with a fluorescence mounting medium. Confocal images were obtained with a BZ-8000 fluorescence microscope equipped with a $10 \mathrm{x}$ eyepiece and a 20x objective lens (Keyence, Osaka, Japan).

Preparation of tumor-bearing mice. The 4T1 cell line is capable of metastasizing to the lungs (19). Cultured 4T1 cells were suspended in cold PBS to result in a density of $1 \times 10^{7}$ cells/ $\mathrm{ml}$. The cells $\left(1 \times 10^{6}\right)$ were injected into the mammary fat pads of an anesthetized mouse. From Day 2 before tumor implantation, SRT1720 (100 mg/kg body weight) or water was given to the mouse 5 times per week. Cisplatin $(6 \mathrm{mg} / \mathrm{kg})$ or sterile saline was injected intraperitoneally on Day 6 after tumor implantation. The size of the tumors was measured on Days 4, $8,12,16$ and 20, using a caliper square along the longer axis (a) and the shorter axis (b). Tumor volume was calculated with the equation $\left[\mathrm{mm}^{3}=\mathrm{ab}^{2} / 2\right]$. After the primary tumor was surgically removed on Day 21 under anesthesia with diethyl ether and thiopental, the surrounding tissues were removed as much as possible. The primary tumors were weighed and frozen at $-20^{\circ} \mathrm{C}$. On Day 28 , the mice were sacrificed and the lungs were intratracheally fixed with a Bouin's solution for $24 \mathrm{~h}$. The number of metastasized tumor colonies $\geq 1 \mathrm{~mm}$ in diameter on the surface of the lungs was counted.

Quantitative RT-PCR. Total RNA was extracted from murine breast cancer primary tumors and cultured cells with an RNeasy Mini kit (Qiagen, Tokyo, Japan) and an Isogen kit (Nippon Gene, Tokyo, Japan), respectively. The RNA samples were reverse transcribed with a PrimeScript RT reagent kit (Takara Bio Inc., Otsu, Japan) followed by amplification with intron spanning primers using a Stratagene MX30000P quantitative PCR machine. PCR primer sequences were: mouse ANGPTL4, 5'-CCTTAACTGTGCCAAGAGCC-3' (forward) and 5'-CTGCAGAGGATAGTAGCGGC-3' (reverse); human ANGPTL4, 5'-TCCTGGACCACAAGCACCTA-3' (forward) and 5'-CTCTCCCCAACCTGGAACAG-3' (reverse); mouse glyceraldehyde phosphate dehydrogenase (GAPDH), 5'-TGA AGCAGCATCTGAGGG-3' (forward) and 5'-CGAAGGTGG 
A

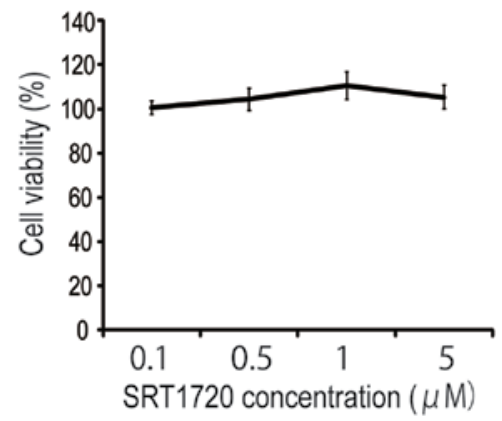

B

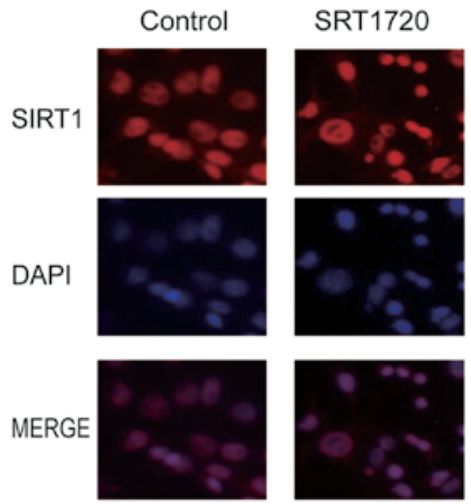

Figure 1. Dose-response of SRT1720 on the viability of 4T1 cells and location of SIRT1 in the presence of SRT1720. (A) 4T1 cells were incubated at varying concentrations of SRT1720. After $24 \mathrm{~h}$, cell viability was determined by an MTS assay (three determinations). (B) 4T1 cells were incubated with or without $5 \mu \mathrm{M}$ SRT1720 for $24 \mathrm{~h}$, reacted with an anti-SIRT1 antibody conjugated with Alexa Fluor 594 and viewed under a confocal laser microscope (upper panels). Nuclei were located by DAPI staining (middle panels). The two panels were merged (lower panels). Magnification, x200.

AAGAGTGGGAG-3' (reverse); human GAPDH, 5'-CGCTC TCTGCTCCTCCTGTT-3' (forward) and 5'-CCATGGTGT CTGAGCGATGT-3' (reverse). The PCR conditions were: denaturation at $95^{\circ} \mathrm{C}$ for $10 \mathrm{~min}$ followed by 40 cycles of denaturation at $95^{\circ} \mathrm{C}$ for $10 \mathrm{sec}$ and extension at $60^{\circ} \mathrm{C}$ for $30 \mathrm{sec}$. The PCR products were quantified by the $2^{-\Delta \Delta \mathrm{Ct}}$ method with GAPDH as an internal control (20).

Enzyme-linked immunosorbent assay (ELISA). The amount of vascular endothelial growth factor (VEGF) in the MDA-MB-231 culture medium was determined with a Quantikine human ELISA kit (R\&D Systems, Minneapolis, MN, USA) according to the manufacturer's instructions Briefly, $50 \mu \mathrm{l}$ of an assay diluent and $200 \mu \mathrm{l}$ of a standard mixture or a sample were transferred to each microplate well which had been coated with a mouse monoclonal anti-VEGF antibody. After incubation for $2 \mathrm{~h}$, the contents of each well were aspirated off, the wall was washed with washing buffer three times, and incubated with $200 \mu \mathrm{l}$ of a polyclonal antibody against VEGF conjugated to horseradish peroxidase for $2 \mathrm{~h}$. After three washes with washing buffer, $200 \mu \mathrm{l}$ of a substrate solution was dispensed into all the wells and shaken for $20 \mathrm{~min}$ at room temperature. The reaction was stopped by adding $50 \mu \mathrm{l}$ of a stop solution, and the color change was measured at $450 \mathrm{~nm}$ in a microplate reader.

Transendothelial cell migration assay. A CytoSelect ${ }^{\mathrm{TM}}$ cell migration assay kit (Cell Biolabs, San Diego, CA, USA) was used for cell migration experiments. Briefly, HUVECs $\left(1 \times 10^{5}\right)$ were seeded into an inner chamber containing $300 \mu \mathrm{l}$ of L-15 culture medium and placed on a well (outer chamber) of a 24 -well plate containing $500 \mu \mathrm{l}$ of the medium. The cells were incubated for 48-72 h until a monolayer formed. Separately, a total of $5 \times 10^{4}$ cells/ml of MDA-MB-231 tumor cells were suspended in a serum-free medium and labeled with a CytoTracker ${ }^{\mathrm{TM}}$ solution for $1 \mathrm{~h}$. Then, $3 \times 10^{4}$ of the labeled cells were plated on each inner chamber whose medium had been replaced with $300 \mu \mathrm{l}$ of L-15 medium containing $2 \mu \mathrm{M}$ SRT1720 and/or $1 \mu \mathrm{g} / \mathrm{ml}$ of cisplatin. These cells were further incubated for $24 \mathrm{~h}$. Non-migrated cells in the inner chamber were removed with cotton-tipped swabs. The cells that migrated outside the inner chamber were collected and resolved in a lysis buffer. The cell lysate was quantified by measuring at $495 \mathrm{~nm}$ (excitation)/520 nm (fluorescence).

Statistical analysis. All data are expressed as the mean \pm SE. Basically, a two-way ANOVA was used to compare multiple groups of data, while differences between two the groups were analyzed with an unpaired Student's t-test. A P-value $<0.05$ was considered statistically significant.

\section{Results}

Effects of SRT1720 on $4 T 1$ cell viability. In this study, SRT1720 was used as a SIRT1 activator for in vitro and in vivo experiments. SRT1720 toxicity has not been previously established for 4T1 breast cancer cells. 4T1 cells at near confluence were incubated with various concentrations of SRT1720 for $24 \mathrm{~h}$. Cell viability was assessed by an MTS assay. It was found that SRT1720 had no effect on cell viability at concentrations between 0.1 and $5 \mu \mathrm{M}$ (Fig. 1A). This result agrees with a previous finding that SRT1720 worked well at a concentration of $0.1 \mu \mathrm{M}$ when human osteosarcoma cells were used (17). Next, we addressed whether SIRT1 exists in the 4T1 cells, and if so, whether SRT1720 alters its abundance or localization. Immunocytochemical images showed appreciable levels of SIRT1 in nuclei of 4T1 cells (Fig. 1B). However, the abundance and localization of SIRT1 did not change after a 24-h incubation in the presence of $1 \mu \mathrm{M}$ SRT1720.

SRT1720 increases lung metastasis of 4T1 cells. 4T1 cells are used as a lung metastasis model of breast cancer. We examined the effect of SRT1720 on the pulmonary metastasis of inoculated 4T1 cells as well as the size and weight of primary tumors. Our previous results showed that the oral administration of SRT1720 to normal mice did not affect body weight or food intake at a dose of $200 \mathrm{mg} / \mathrm{kg}$ over a period of 70 days (18). Therefore, SRT1720 was given at a dose of $100 \mathrm{mg} / \mathrm{kg}$ body weight. Cisplatin is one of the most popular anticancer drugs and clinically used for the treatment of various types 
A

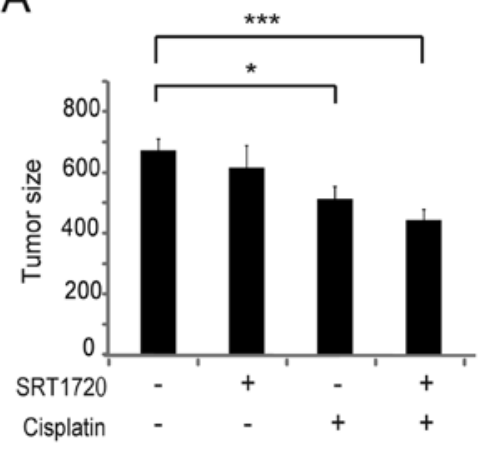

B

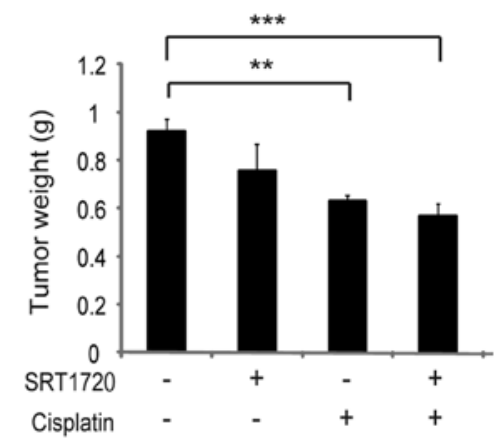

C

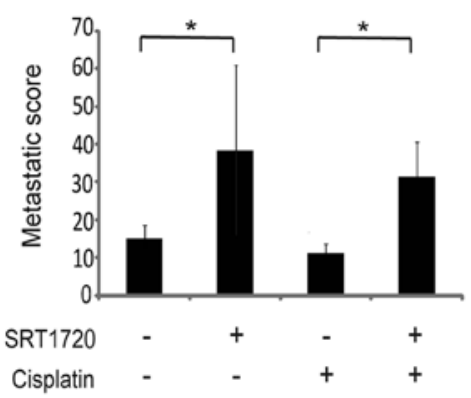

Figure 2. Size and weight of primary tumors in mice xenografted with 4T1 cells, and the number of metastatic tumors in the lungs, when mice were treated with SRT1720, and/or cisplatin. (A) Average size of primary tumors in a breast cancer model on Day 20 after implantation. Tumor volume was calculated using the following formula: tumor volume $\left(\mathrm{mm}^{3}\right)=\mathrm{ab}^{2} / 2$, as described in Materials and methods. (B) Average weight of primary tumors in the breast cancer model. Tumor volume was determined as described in Materials and methods. (C) Metastatic lung tumors were counted 28 days after implantation. The number of tumors $1 \mathrm{~mm}$ or more in diameter was counted. The results are shown as the mean \pm SEM for three independent groups. ${ }^{* * *} \mathrm{P}<0.001 ;{ }^{* *} \mathrm{P}<0.01 ;{ }^{*} \mathrm{P}<0.05$.

of cancer. This drug was injected once into mice on Day 6 after the implantation of 4T1 cells. The primary tumors were removed on Day 21 and measured. On Day 28, colonies on the surface of the lungs of the sacrificed mice were counted. As expected, primary tumor size and weight were significantly decreased in the cisplatin group (Fig. 2A and B). SRT1720 alone had no effect on these values, and actually slightly stimulated the cisplatin-dependent reduction in tumor size and weight. However, when metastatic foci on the lungs were counted, SRT1720 was found to increase lung metastasis by
A ANGPTL4 mRNA (primary tumors)

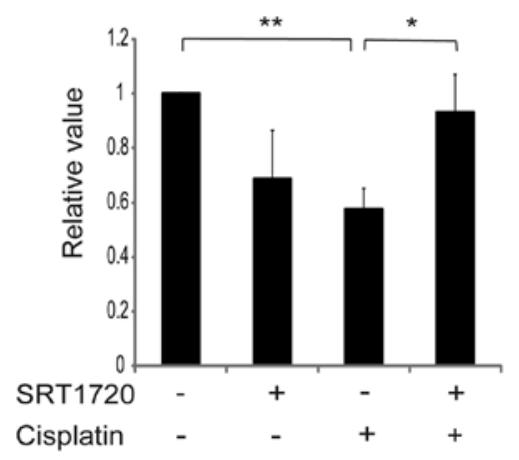

B ANGPTL4 mRNA (4T1 cells)

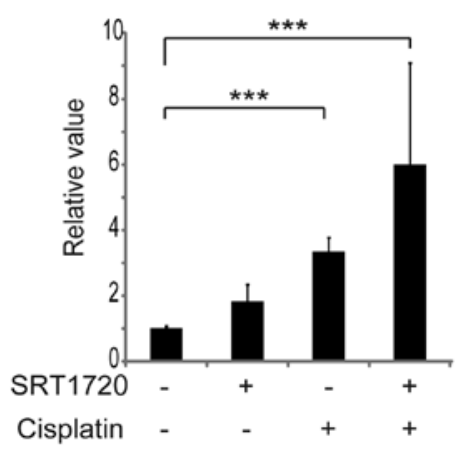

Figure 3. Effects of SRT1720 and/or cisplatin on ANGPTL4 mRNA levels in primary tumors and cultured 4T1 cells. SRT1720 and/or cisplatin mediated the expression of genes promoting the lung metastasis of breast tumors. (A) ANGPTL4 mRNA levels in the primary tumors were determined by quantitative RT-PCR as described in Materials and methods. GAPDH was used as an internal control. Data are shown as the mean \pm SE of three independent experiments. ${ }^{* *} \mathrm{P}<0.01 ;{ }^{*} \mathrm{P}<0.05$. (B) The ANGPTL4 mRNA level in $4 \mathrm{~T} 1$ cells cultured with SRT1720 and/or cisplatin. ${ }^{* * *} \mathrm{P}<0.001$.

about $150 \%$, even in the presence of cisplatin (Fig. 2C). These results show that SRT1720 does not substantially affect the size and weight of primary tumors, but dramatically increases lung metastasis. Cisplatin itself failed to prevent the metastasis.

Effects of SRT1720 on ANGPTL4 mRNA levels in the primary tumor and $4 T 1$ cells. The expression of at least 18 genes is elevated during breast-to-lung metastasis $(4,5)$. It is of interest to see whether the expression of these genes fluctuated during metastasis in mice treated with SRT1720. Here, we focused on the ANGPTL4 gene because its product is considered to be involved in metastasis through the stimulation of angiogenesis (4). The gene expression of ANGPTL4 was evaluated by quantitative RT-PCR with total RNA from the primary tumor and original 4T1 cells. As expected, cisplatin reduced ANGPTL4 mRNA levels in the primary tumor tissues, but this reduction was cancelled at the control level by SRT1720 (Fig. 3A). This result is in excellent agreement with the general action of cisplatin as an antitumor agent. Although it was unexpected that SRT1720 alone had no effect in elevating the ANGPTL4 mRNA level, SRT1720 abolished the effectiveness of cisplatin. This result supports the conclusion of this study that SRT1720 aggravates the metastasis. On the other hand, when we examined the ANGPTL4 mRNA levels 
A ANGPTL4 mRNA (MDA-MB231 cells)

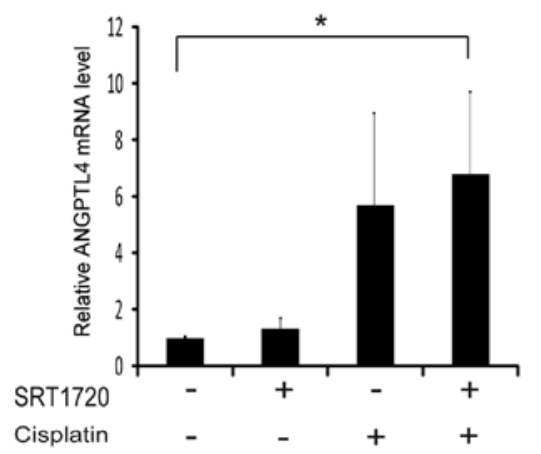

B VEGF (MDA-MB231 cells)

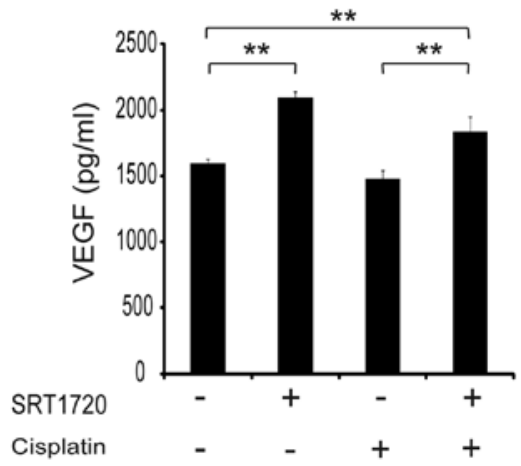

Figure 4. ANGPTL4 mRNA and VEGF levels in human breast cancer. MDA-MB-231 cells cultured with SRT1720 and/or cisplatin. (A) MDA-MB-231 cells were incubated with SRT1720 and/or cisplatin for $24 \mathrm{~h}$, and ANGPTL4 mRNA levels were determined by quantitative RT-PCR. GAPDH was used as an internal control. (B) After $24 \mathrm{~h}$ of exposure to SRT1720 and/or cisplatin, the VEGF level in the medium of MDA-MB-231 cells was measured by ELISA. ${ }^{* *} \mathrm{P}<0.01 ;{ }^{*} \mathrm{P}<0.05$.

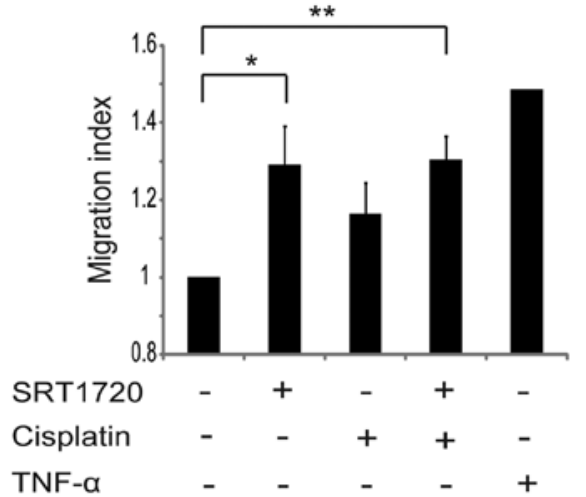

Figure 5. Effects of SRT1720 and/or cisplatin on the migration of MDA-MB-231 cells across a HUVEC layer. The migration assay was carried out as described in Materials and methods. The cells that migrated across a HUVEC layer that had been labeled with fluorescent materials were harvested and lysed cells were quantified by measuring the fluorescence. TNF- $\alpha$ $(1 \mathrm{ng} / \mathrm{ml})$ was used as a positive control. ${ }^{* *} \mathrm{P}<0.01 ;{ }^{*} \mathrm{P}<0.05$

in the cultured 4T1 cells, the result was largely different from that for the primary tumors. Cisplatin and SRT1720 solely tended to increase ANGPTL4 mRNA levels, but when used in combination, the level was increased 5-fold (Fig. 3B). This discrepancy might be due to the difference between in vivo and in vitro experiments. However, both experiments showed that SRT1720 increased ANGPTL4 mRNA levels compared with cisplatin alone.

Effects of SRT1720 on ANGPTL4 and VEGF levels in human breast cancer cells. The results above indicate the possibility that SRT1720 aggravates the inhibition of metastasis by cisplatin. In other words, SRT1720 may be harmful to the treatment of breast-to-lung cancer metastasis with cisplatin.The final aim of our study is to know whether the results obtained with animal experiments are applicable to humans. Thus, we attempted the same experiment with MDA-MB-231 human breast cancer cells. SRT1720 was not toxic to MDA-MB-231 cells when used below a concentration of $5 \mu \mathrm{M}$ (data not shown). Similar to the result for 4T1 cells, cisplatin but not SRT1720 increased
ANGPTL4 mRNA levels by $75 \%$, and SRT1720 did not inhibit the action of cisplatin, suggesting that, in the presence of cisplatin, murine 4T1 cells resemble human MDA-MB-231 cells with respect to ANGPTL4 expression (Fig. 4A). Next, we examined VEGF secretion which is considered to be crucial for angiogenesis. MDA-MB-231 cells were treated with SRT1720, cisplatin or both, and the concentration of VEGF in the culture medium was determined by ELISA (Fig. 4B). In this case, SRT1720 increased the VEGF concentration by about $30 \%$ relative to the control. Cisplatin had no effect, but SRT1720 was still effective even in the presence of cisplatin.

SRT1720 promotes MDA-MB-231 tumor cell migration across a HUVEC layer. Since we found that SRT1720 increases the secretion of VEGF in human cancer cells, we addressed whether SRT1720 indeed stimulates the migration of these cells. For this purpose, a transendothelial migration assay using a HUVEC monolayer was carried out where TNF- $\alpha$ was used as a positive control (21). Under these conditions, TNF- $\alpha$ indeed stimulated the migration of MDA-MB-231 cells across the layer by $50 \%$, whereas SRT1720 increased the migration over the untreated control by $29 \%$. Cisplatin failed to suppress the migration stimulated by SRT1720 (Fig. 5). These results strongly indicate that SRT1720 can promote the migration of breast cancer cells across the HUVEC monolayer, and cisplatin is not related to this event.

\section{Discussion}

In the present study, we have provided evidence that SRT1720 promotes lung metastasis of implanted murine breast cancer cells, and is effective in the stimulation of VEGF excretion and cell migration found among human cancer cells.

Murine breast cancer 4T1 cells possess strong metastatic potential, but it had not previously been determined whether these cells contain SIRT1. Fig. 1 shows that the SIRT1 protein is present in nuclei, and treatment with SRT1720 alters neither the protein level nor its localization. SRT1720 binds to an allosteric site, which is situated on the amino terminal side of the catalytic domain, and an effector-SIRT1-acetylated substrate 
tertiary complex causes a conformational change to enhance the deacetylase activity (17). Thus, SRT1720 is considered not to increase the SIRT1 protein level directly, for example by a feed-forward mechanism. Moreover, it is difficult to present evidence of whether SRT1720 actually activates SIRT1 deacetylase activity in vivo.

Although SIRT1 is considered to have beneficial roles in a variety of physiological phenomena, its actions in cancer cells seem to be contradictory; SIRT1 acts as a tumor suppressor $(22,23)$ and as a tumor promoter $(10,24)$. The reason for this remains unclear. SIRT1 activators sometimes act as antioxidating molecules in addition to the original deacetylase (25). SIRT1 deacetylates not only histone proteins but various transcription factors such as p53, the forkhead box class $\mathrm{O}$ (FOXO) transcription factor, peroxisome proliferator activated receptors (PPARs) co-activator $1 \alpha(\mathrm{PGC}-1 \alpha)$ and nuclear factor- $\kappa \mathrm{B}(10,26)$. The substrates of SIRT1 are expected to differ from one organ to another (27). Therefore, SIRT1 would play different roles depending on the type of tissue or tumor, although SIRT1 expression is upregulated in a variety of cancer types as mentioned in the Introduction.

The process of tumor metastasis is very complicated. Several discrete steps can be discerned in the course of metastasis; tumor invasion, intravasation into vessels, extravasation from vessels, and penetration and growth in target organs (28). While the genes for ANGPTL4, epidermal growth factor receptor ligand epiregulin, prostaglandinendoperoxide synthase 2, matrix metalloproteinase-1, are thought to be important for lung metastasis, ANGPTL4 functions at the steps of intravasation and extravasation (4). Therefore, we focused on ANGPTL4. Interestingly, in the primary tumor cells, cisplatin significantly suppressed the ANGPTL4 mRNA levels as expected, but this action was overcome by SRT1720. To our knowledge, there is no report of a SIRT1 and ANGPTL4 relationship. ANGPTL4 was initially found in the adipose tissue as a novel target of peroxisome proliferator-activated receptor $\alpha$ (PPAR $\alpha$ ), which encodes a secreted and fasting-induced adipose factor (29). Purushotham et al (30) showed that the hepatic SIRT1 upregulates the expression of PPAR $\alpha$. Since Suchanek et al (31) reported the occurrence of PPAR $\alpha$ in the human breast cancer cell lines MDA-MB-231 and MCF-7, it is tempting to speculate that SRT1720 can enhance ANGPTL4 gene expression by activating PPAR $\alpha$.

The findings in Figs. 2 and 3 led us to suppose that SRT1720 enhances the potential of cisplatin. We considered that intravasation and/or extravasation might be key steps in SIRT1-enhanced lung metastasis, because SRT1720 had no effect on the number or mass of primary tumors. We focused on VEGF, one of the most important factors for tumor metastasis, promoting tumor angiogenesis, invasion and cell migration $(32,33)$. We found that SRT1720 enhanced VEGF production in MDA-MB-231 cells irrespective of cisplatin (Fig. 4B). Arany et al (34) reported that PGC-1 $\alpha$ regulates VEGF expression and angiogenesis in an HIF-dependent manner. PGC-1 $\alpha$ is a major co-activator of SIRT1 (35). Thus, it is possible that SIRT1 enhances VEGF levels via PGC-1 $\alpha$ activation. Potente et al (36) reported that SIRT1 regulates endothelial angiogenic functions by modulating the transcriptional activity of FOXO1, which is regulated by SIRT1 through its deacetylation (37). Furthermore, Gealekman et al (38) showed that the simultaneous production of VEGF and ANGPTL4 enhances angiogenesis in adipocytes. Therefore, it is tempting to assume that SRT1720 increases ANGPTL4 and VEGF levels by activating several nuclear factors, thereby promoting metastasis through enhanced angiogenesis. A series of invasion steps are regulated by multiple factors such as FOXO1 and PGC-1 $\alpha$ through the deacetylating activity of SIRT1 (39).

In conclusion, the present findings imply that SIRT1 promotes lung metastasis by facilitating tumor cell migration across a vascular endothelial cell layer. SIRT1 is well known as a longevity gene, and its activators such as resveratrol are considered to be promising agents for age-related diseases. However, the present results, along with the upregulation of SIRT1 expression in various tumors, suggest SIRT1 activators to be harmful when applied to patients with malignant diseases. A reverse study is needed to clarify whether SIRT1 inhibitors actually prevent the lung metastasis of breast cancer cells. Such attempts are now underway in our laboratory.

\section{Acknowledgements}

We thank Dr Hiroki Inoue, Dr Yukiko Kanatani and Dr Yu Yamazaki, Mrs. Kazuko Hayashi and Tomomi Kubo for their excellent technical assistance.

\section{References}

1. Perret GY and Crepin M: New pharmacological strategies against metastatic spread. Fundam Clin Pharmacol 22: 465-492, 2008.

2. Gupta GP, Nguyen DX, Chiang AC, et al: Mediators of vascular remodelling co-opted for sequential steps in lung metastasis. Nature 446: 765-770, 2007.

3. Steeg PS: Tumor metastasis: mechanistic insights and clinical challenges. Nat Med 12: 895-904, 2006.

4. Chiang AC and Massague J: Molecular basis of metastasis. N Engl J Med 359: 2814-2823, 2008.

5. Minn AJ, Gupta GP, Siegel PM, et al: Genes that mediate breast cancer metastasis to lung. Nature 436: 518-524, 2005.

6. Blander G and Guarente L: The Sir2 family of protein deacetylases. Ann Rev Biochem 73: 417-435, 2004.

7. Kaeberlein M, McVey M and Guarente L: The SIR2/3/4 complex and SIR2 alone promote longevity in Saccharomyces cerevisiae by two different mechanisms. Genes Dev 13: 2570-2580, 1999.

8. Bordone L and Guarente L: Calorie restriction, SIRT1 and metabolism: understanding longevity. Nat Rev Mol Cell Biol 6: 298-305, 2005.

9. Lagouge M, Argmann C, Gerhart-Hines Z, et al: Resveratrol improves mitochondrial function and protects against metabolic disease by activating SIRT1 and PGC-1alpha. Cell 127: 1109-1122, 2006.

10. Liu T, Liu PY and Marshall GM: The critical role of the class III histone deacetylase SIRT1 in cancer. Cancer Res 69: 1702-1705, 2009.

11. Kojima K, Ohhashi R, Fujita Y, et al: A role for SIRT1 in cell growth and chemoresistance in prostate cancer PC3 and DU145 cells. Biochem Biophys Res Commun 373: 423-428, 2008.

12. Ota H, Tokunaga E, Chang K, et al: Sirt1 inhibitor, Sirtinol, induces senescence-like growth arrest with attenuated Ras-MAPK signaling in human cancer cells. Oncogene 25: 176-185, 2006

13. Heltweg B, Gatbonton T, Schuler AD, et al: Antitumor activity of a small-molecule inhibitor of human silent information regulator 2 enzymes. Cancer Res 66: 4368-4377, 2006.

14. Lain S, Hollick JJ, Campbell J, et al: Discovery, in vivo activity, and mechanism of action of a small-molecule p53 activator. Cancer Cell 13: 454-463, 2008. 
15. Bishayee A: Cancer prevention and treatment with resveratrol: from rodent studies to clinical trials. Cancer Prev Res 2: 409-418, 2009.

16. Knutson MD and Leeuwenburgh C: Resveratrol and novel potent activators of SIRT1: effects on aging and age-related diseases. Nutr Rev 66: 591-596, 2008.

17. Milne JC, Lambert PD, Schenk S, et al: Small molecule activators of SIRT1 as therapeutics for the treatment of type 2 diabetes. Nature 450: 712-716, 2007.

18. Yamazaki Y, Usui I, Kanatani Y, et al: Treatment with SRT1720, a SIRT1 activator, ameliorates fatty liver with reduced expression of lipogenic enzymes in MSG mice. Am J Physiol Endocrinol Metab 297: E1179-E1186, 2009.

19. Pidgeon GP, Harmey JH, Kay E, Da Costa M, Redmond HP and Bouchier-Hayes DJ: The role of endotoxin/lipopolysaccharide in surgically induced tumour growth in a murine model of metastatic disease. Br J Cancer 81: 1311-1317, 1999.

20. Livak KJ and Schmittgen TD: Analysis of relative gene expression data using real-time quantitative PCR and the 2(-Delta Delta C(T)) method. Methods 25: 402-408, 2001.

21. Xu Z, Vonlaufen A, Phillips PA, et al: Role of pancreatic stellate cells in pancreatic cancer metastasis. Am J Pathol 177: 2585-2596, 2010.

22. Deng CX: SIRT1, is it a tumor promoter or tumor suppressor? Int J Biol Sci 5: 147-152, 2009.

23. Saunders LR and Verdin E: Sirtuins: critical regulators at the crossroads between cancer and aging. Oncogene 26: 5489-5504, 2007.

24. Lim CS: SIRT1: tumor promoter or tumor suppressor? Med Hypotheses 67: 341-344, 2006.

25. Bosch-Presegue L, Raurell-Vila H, Marazuela-Duque A, et al: Stabilization of Suv39H1 by SirT1 is part of oxidative stress response and ensures genome protection. Mol Cell 42: 210-223, 2011.

26. Zschoernig B and Mahlknecht U: SIRTUIN 1: regulating the regulator. Biochem Biophys Res Commun 376: 251-255, 2008.

27. Zeng L, Chen R, Liang F, et al: Silent information regulator, Sirtuin 1, and age-related diseases. Geriatr Gerontol Int 9: 7-15, 2009.

28. Gupta GP and Massague J: Cancer metastasis: building a framework. Cell 127: 679-695, 2006.
29. Kersten S, Mandard S, Tan NS, et al: Characterization of the fasting-induced adipose factor FIAF, a novel peroxisome proliferator-activated receptor target gene. J Biol Chem 275: 28488-28493, 2000

30. Purushotham A, Schug TT, Xu Q, Surapureddi S, Guo X and Li X: Hepatocyte-specific deletion of SIRT1 alters fatty acid metabolism and results in hepatic steatosis and inflammation. Cell Metab 9: 327-338, 2009.

31. Suchanek KM, May FJ, Robinson JA, Lee WJ, Holman NA, Monteith GR and Roberts-Thomson SJ: Peroxisome proliferatoractivated receptor alpha in the human breast cancer cell lines MCF-7 and MDA-MB-231. Mol Carcinog 34: 165-171, 2002.

32. Linderholm B, Lindh B, Tavelin B, Grankvist K and Henriksson R: p53 and vascular-endothelial-growth-factor (VEGF) expression predicts outcome in 833 patients with primary breast carcinoma. Int J Cancer 89: 51-62, 2000.

33. Mercurio AM, Lipscomb EA and Bachelder RE: Non-angiogenic functions of VEGF in breast cancer. J Mammary Gland Biol Neoplasia 10: 283-290, 2005.

34. Arany Z, Foo SY, Ma Y, et al: HIF-independent regulation of VEGF and angiogenesis by the transcriptional coactivator PGC-1alpha. Nature 451: 1008-1012, 2008.

35. Rodgers JT, Lerin C, Haas W, Gygi SP, Spiegelman BM and Puigserver P: Nutrient control of glucose homeostasis through a complex of PGC-1alpha and SIRT1. Nature 434: 113-118, 2005.

36. Potente M, Ghaeni L, Baldessari D, et al: SIRT1 controls endothelial angiogenic functions during vascular growth. Genes Dev 21: 2644-2658, 2007.

37. Jiang WJ: Sirtuins: novel targets for metabolic disease in drug development. Biochem Biophys Res Commun 373: 341-344, 2008.

38. Gealekman O, Burkart A, Chouinard M, Nicoloro SM, Straubhaar J and Corvera S: Enhanced angiogenesis in obesity and in response to PPARgamma activators through adipocyte VEGF and ANGPTL4 production. Am J Physiol Endocrinol Metab 295: E1056-E1064, 2008.

39. Morris BJ: A forkhead in the road to longevity: the molecular basis of lifespan becomes clearer. J Hypertens 23: 1285-1309, 2005. 